\title{
Oportunidades de aplicación de las tecnologías de la información y comunicación (TICS) para fortalecer la lucha contra la anemia en Perú
}

\section{Opportunities for using information and communication technology (ICT) for strengthening campaigns against anemia in Peru}

Correspondencia

Elizabeth Espinoza-Portilla espinoza.portilla@gmail.com

Recibido: 08/01/2019

Arbitrado por pares

Aprobado: 27/03/2019

Citar como: Espinoza-Portilla E, Henríquez-Suárez M, VillanuevaCadenas GJ. Oportunidades de aplicación de las tecnologías de la información y comunicación (TICS) para fortalecer la lucha contra la anemia en Perú. Acta Med Peru. 2019;36(2):152-6
Elizabeth Espinoza-Portilla1,a, Milagro Henríquez-Suárez 2,b, Gladis J.

Villanueva-Cadenas ${ }^{2, c}$

1 Universidad ESAN. Lima, Perú.

2 Universidad Nacional José Faustino Sánchez Carrión. Huacho, Perú.

a Médico-cirujano, Magíster en Estudios de Políticas Públicas, Magíster en Demografía y Población, Doctora en Gobierno y Políticas Públicas, b Licenciada en Trabajo Social, Magíster en Salud Pública, Doctora en Administración, c Licenciada en Enfermería, Magíster en Ciencias de la Educación Superior.

\section{RESUMEN}

La anemia es un grave problema de salud pública en América Latina. En el Perú la proporción de niños entre 6 y 35 meses con anemia fue de 43,5\% en el 2018. El objetivo de esta publicación es revisar las diferentes oportunidades de aplicación de las tecnologías de información y comunicación (TICS) en el fortalecimiento de la lucha contra la anemia en nuestro país; bajo la premisa de que la aplicación de las TICs puede convertirse en una excelente oportunidad para mejorar el acceso y calidad de los servicios de salud ofrecidos por el sistema público. La literatura revisada muestra diversas experiencias previas que incluyen la utilización de mensajes de texto a través de celulares y desarrollo de aplicativos para teléfonos inteligentes. Sin embargo, se requiere de más investigación, con mejores niveles de evidencias que, finalmente, ayuden a la elaboración de estrategias eficientes y a la mejora de políticas sanitarias en un tema que es una prioridad nacional de cara al bicentenario.

Palabras clave: Anemia; Tecnologías de información; Teléfono celular; Mensaje de texto; Salud pública (fuente: DeCS BIREME). 


\begin{abstract}
Anemia is a serious public health problem in Latin America. In Peru, the rate of 6- to 35- month old children with anemia was $43.5 \%$ in 2018. The aim of this publication is to review the different opportunities for using information and communication technology (ICT) aiming to strengthen campaigns against anemia in Peru. This concept is based on the idea stating that the use of ICT may become an excellent opportunity for improving both access to and quality of public Peruvian healthcare services. The reviewed literature shows many previous experiences, including smartphone text messages (SMS) and the development of apps for smartphones. However, more research is needed, with better evidence level; which finally may help to design efficient strategies and to improve public health policies in a topic that is a national priority for the forthcoming independence bicentennial.
\end{abstract}

Keywords: Anemia; Information technology; Cellular phone; Text messaging; Public health (source: MeSH NLM).

\section{INTRODUCCIÓN}

La anemia es un grave problema de salud pública en América Latina. Según el Banco Mundial, el porcentaje de niños menores de 5 años con anemia en América Latina y el Caribe fue de $28 \%$ en el 2016. Si bien esta cifra muestra una reducción significativa con respecto al 40\% reportado en el 1990 , persisten desigualdades entre los países de la región ${ }^{[1]}$.

En nuestro país, según lo encontrado por la Encuesta Demográfica y de Salud Familiar (ENDES), desarrollada durante el año 2018, la proporción de niños entre 6 y 35 meses, a nivel nacional, con anemia fue de $43,5 \%{ }^{[2]}$. Ante esta situación, se hace necesario implementar políticas multisectoriales e intergubernamentales que enfrenten esta problemática de manera innovadora. Estas políticas deben incluir una estrategia de comunicación integral que permita a la población tener más información sobre este problema de salud pública, con el fin de identificar oportunamente y tratar apropiadamente a la persona con anemia. Es así que, en julio del 2018, el Gobierno publicó el Plan Multisectorial de Lucha contra la Anemia que refleja el esfuerzo multisectorial, con enfoque de gestión territorial, de manera articulada, que plantea la meta de reducción de la anemia en niños menores de 3 años al 19\% para el año $2021^{[3]}$.

Por otro lado, en los últimos años el aumento del uso de los teléfonos celulares y la Internet han facilitado nuevas formas de comunicación masiva y de interacción con los ciudadanos ${ }^{[4]}$. Según los resultados del Censo Nacional 2017: XII de Población y VII de Vivienda del Instituto Nacional de Estadística e Informática (INEI), 83,8\% de los hogares cuentan con un celular (6 912745 hogares), cifra que se incrementó significativamente con respecto al censo nacional realizado en el 2007 (42,9\% que representó 2898406 hogares). En forma similar, con respecto al acceso a Internet, el INEI reportó que 2314182 hogares (28,0\%) cuentan con acceso, de acuerdo a lo reportado en el censo nacional 2017; a diferencia del realizado 10 años antes, donde solo se encontró a 458158 hogares (6.8\%) con acceso a este recurso ${ }^{[5,6]}$.

En este contexto, las tecnologías de información y comunicación (TICS) se convierten en una opción de mucha utilidad para mejorar el manejo y control de prioridades sanitarias, como la anemia, en el marco de las estrategias de salud pública desplegadas por el Estado. Se conoce que las TICs permiten mejorar el acceso y calidad de los servicios de salud, incrementan la efectividad de los programas de salud pública y pueden reducir los costos de la asistencia sanitaria ${ }^{[7,8]}$. Cuando se aprovechan estas utilidades para buscar una mejor gestión de la información (almacenamiento, recuperación y el uso óptimo de los datos), y así mejorar la toma de decisiones en el área biomédica, se puede hablar de la aplicación de informática biomédica ${ }^{\left[{ }^{[8}\right.}$.

La informática biomédica tiene muchas aplicaciones que se pueden implementar en el marco de la lucha contra la anemia; por ejemplo: la implementación de historias clínicas electrónicas que registren y monitoricen a los niños con anemia, los sistemas de información de laboratorio clínico que registren los resultados de hemoglobina, las tecnologías móviles para el envío de mensajes de texto y registros móviles de información, entre otros ${ }^{[7]}$. El objetivo de esta publicación es revisar las diferentes oportunidades de aplicación de las TICs para fortalecer la lucha contra la anemia.

\section{Promoción de la salud a través de mensajes usando las TICs}

Existen estudios y evaluaciones que muestran el potencial de las intervenciones con tecnologías móviles para mejorar la salud infantil y salud materna en países en vías en desarrollo, pero la evidencia es aún limitada ${ }^{[9]}$. Sin embargo, en el caso de mensajes de texto, se tiene alguna evidencia de que mejoran la adherencia al tratamiento y la asistencia a las citas médicas en diversas condiciones de salud a través de recordatorios.

La revisión sistemática de Kannisto et al. evalúa el impacto del uso de recordatorios usando mensajes de texto a través de celulares (short message service o SMS) sobre la asistencia a consultas y la adherencia al tratamiento. Se encontró un aumento de niveles de adherencia al tratamiento (40\% de estudios analizados), aumento de la asistencia a citas médicas (18\%) y disminución de la incidencia de cancelaciones (18\%). Se tuvo como otros impactos positivos como la reducción de dosis no tomadas, 
actitudes más positivas hacia la medicación y reducción en la tasa de interrupciones del tratamiento ${ }^{[10]}$.

Por otro lado, la revisión sistemática sobre salud móvil de Lee et al. que evalúa la efectividad de las intervenciones móviles en salud sobre la salud materna, neonatal e infantil en países de ingresos bajos y medios; en el cual, a diferencia del estudio anterior, si se incluye el análisis de la entrega de mensajes de texto sobre temas de nutrición ${ }^{[11]}$. Se encontró que la incidencia de lactancia materna exclusiva en el grupo que recibió mensajes de texto informativos aumentó en los tres estudios analizados en comparación al grupo que no recibió mensajes de texto. Adicionalmente, en dos de los estudios analizados, aumentaron las tasas de asistencia a los controles prenatales y de crecimiento y desarrollo en comparación al grupo que no recibió los mensajes recordatorios.

Si bien no se encontraron estudios en Latinoamérica que evalúen específicamente el impacto del envío de SMS en el tema específico de reducción de anemia, si se ha encontrado evidencia proveniente de países asiáticos y africanos, relativa a intervenciones que utilizan SMS para mejorar la adherencia al tratamiento con micronutrientes en niños o gestantes. A continuación, explicaremos algunos ejemplos resaltantes.

Duan et al. ${ }^{[11]}$ evalúan el impacto de los mensajes de texto diarios en la incidencia de anemia y el crecimiento infantil en niños de 6 meses, en un centro de salud en Mongolia. Se realizó el envío de mensajes de texto a los celulares de mujeres gestantes a pocas semanas de dar a luz. Estos mensajes contenían información sobre cómo lograr una adecuada nutrición infantil. A los 6 meses, se realizó el seguimiento a ambos grupos para evaluar el estado nutricional de los bebés nacidos luego de la intervención y se halló que la prevalencia de anemia $(p<0,01)$ y sobrepeso y obesidad $(p=0,02)$ era menor en el grupo de tratamiento con respecto al grupo control ${ }^{[12]}$.

Un estudio similar, de Zhou et al. evalúa la adherencia al tratamiento con micronutrientes en niños entre 6 a 12 meses en China. Se asignó a la población de interés en tres grupos diferenciados: a) Entrega libre del suplemento, b) Entrega del suplemento y recordatorio diario mediante mensaje de texto y $\mathrm{c}$ ) Grupo control. Se encontró que el grupo que recibió los mensajes de texto tuvo un incremento en los niveles de adherencia al tratamiento (efecto marginal $=0,10$; IC $95 \%=0,03$ a 0,16 ) comparado con el grupo que sólo recibió suplementos. Asimismo, se encontró que el grupo que recibió SMS tuvo una disminución de la incidencia de anemia con respecto al grupo control (efecto marginal $-0,07$; IC $95 \%=-0,12$ a $-0,01)$, pero no con respecto al grupo que sólo recibió el suplemento (efecto marginal = - 0,03 ; IC $95 \%=-0,09$ a 0,03$)^{[13]}$.

En otro estudio, Khorshid et al. examinaron el efecto de mensajes de texto, en una intervención de 12 semanas, para mejorar la adherencia en la toma de suplementos de hierro en mujeres gestantes en Irán. Si bien encontraron mejoras en la adherencia (en comparación al grupo de control que no recibió los recordatorios de SMS), no se demostraron diferencias significativas en los niveles de hierro sérico ${ }^{[14]}$.

Rounseville et al. evaluaron el impacto de mensajes de texto para promocionar cambios comportamentales de cuidadores de niños menores de 6 años en Ecuador. Estos mensajes de texto buscaban incentivar la asistencia al centro de salud, el consumo de suplemento de hierro, y brindar información nutricional y sobre prácticas de agua saneamiento e higiene. La intervención con este programa resultó en mejoras en indicadores como el peso por talla; y en la disminución de la frecuencia de enfermedades en los niños ${ }^{[15]}$.

En el contexto local, se encontró una nota metodológica elaborada por el Ministerio de Desarrollo e Inclusión Social del Perú (MIDIS) para un piloto de intervención que usa la mensajería de texto para promover el cambio de comportamiento en gestantes y cuidadores de niños menores de 3 años con el objetivo de prevenir y disminuir la anemia en el ámbito del Programa JUNTOS ${ }^{[16]}$. Sin embargo, aún no se han reportado los resultados de esta iniciativa o el posible impacto que ha tenido en las cifras de anemia, en las familias intervenidas.

La evidencia encontrada sugiere que el uso de mensajes de texto puede ser útil para mejorar el estado nutricional y el crecimiento de los niños mediante una intervención temprana. Adicionalmente, puede incrementar la adherencia al tratamiento planteado o a medidas de salud pública que hayan probado eficacia en la disminución de la incidencia de anemia.

\section{Uso de Apps para teléfonos inteligentes en la lucha contra la anemia}

Se han encontrado pocas experiencias internacionales sobre el desarrollo de Apps especializadas para la detección y lucha contra la anemia, sobre todo en países de medianos-bajos ingresos económicos. Se puede destacar la publicación de Mannino et al. de la Universidad Emory de Atlanta - Estados Unidos, quienes desarrollaron un App para teléfonos inteligentes que con sólo una foto de las uñas (además de otras partes del cuerpo, como la palma de la mano o la lengua) podía medir y detectar la probabilidad de presentar anemia, gracias a un algoritmo que calcula los niveles de hemoglobina en la sangre analizando el color de la piel y otros datos técnicos. Esta evaluación fue realizada en cien personas, y aún se encuentra en discusión la capacidad de esta nueva herramienta de sustituir al tradicional análisis de sangre "in situ"; sin embargo, puede convertirse en una potencial solución para lugares con alta incidencia de anemia que carecen de equipos especializados y personal capacitado ${ }^{[17]}$.

En nuestro país, el Ministerio de Salud del Perú (MINSA) reportó el desarrollo de una aplicación para teléfonos inteligentes con sistema Android denominada "App de Lucha y Movilización contra la Anemia - ALMA" que brinda a los padres de familia información personalizada por cada uno de sus hijos (menores de 36 meses) para prevenir y controlar esta enfermedad ${ }^{[18]}$. El reporte encontrado menciona que este aplicativo móvil 
permite hacer el seguimiento de las fechas clave para el control de crecimiento de los hijos, y las fechas de los controles de hemoglobina, mediante el uso de recordatorios. Además, ofrece recetas para preparar alimentos ricos en hierro de procedencia animal para prevenir la anemia ${ }^{[19]}$.

\section{Oportunidades de aplicación local de TICs en el contexto peruano}

Los estudios presentados en este artículo demuestran las múltiples oportunidades que ofrecen las TICs para fortalecer la lucha contra la anemia. En especial, las tecnologías móviles permiten ofrecer una amplia gama de servicios, desde el uso de mensajes de texto para enviar información clara y relevante para padres y profesionales de salud hasta el uso de teléfonos inteligentes para colecta de datos; por ende se pueden convertir en un valioso instrumento para optimizar la toma de decisiones ${ }^{[8]}$.

Una de las ventajas de uso de las TICs es su diverso ámbito de aplicación, sobre todo en un país como el nuestro, se pueden utilizar en áreas urbanas como en áreas rurales, lo que se convierte en una oportunidad de llegar a poblaciones alejadas o de difícil acceso. Por ejemplo, Breslauer et al. de la Universidad de California-Berkeley, desarrollaron un microscopio óptico montado en un teléfono móvil y demostraron su potencial uso clínico para la detección de anemia falciforme, una enfermedad que es prevalente en países de bajos ingresos ${ }^{[20]}$. Bajo el mismo principio, iniciativas como estas podrían ayudar a la detección temprana de anemia por deficiencia de hierro en establecimientos de salud de primer nivel, en donde a veces la deficiencia de equipamiento dificulta la labor preventivapromocional del personal asistencial.

Adicionalmente, las TICs podrían ser un elemento clave para la optimización del seguimiento y evaluación del Plan Multisectorial de Lucha contra la Anemia. Por ejemplo, la implementación de registros electrónicos para monitorizar las actividades y avances de las instancias de articulación local y las instancias de articulación regional, podría terminar entregando información sustancial para el desarrollo de un observatorio multisectorial de anemia; el cual no sólo es un instrumento para mostrar los avances de los indicadores y actividades, sino que debe constituirse en un instrumento de gestión para las autoridades a nivel local, regional y nacional.

En este contexto, se debe destacar un proyecto en curso, impulsado desde el laboratorio de innovación Ayni Lab Social del Ministerio de Desarrollo e Inclusión Social, denominado "Sistema portátil para el diagnóstico de anemia basado en el análisis de la conjuntiva ocular usando un smartphone e inteligencia artificial" el cual es liderado por la Universidad Peruana Cayetano Heredia y actualmente en período de ejecución ${ }^{[21,22]}$. Si los resultados de este proyecto no invasivo para detectar anemia en niños son alentadores, se debería evaluar el escalamiento a nivel nacional, ya que se evitarían procedimientos invasivos en los niños y representaría una excelente oportunidad de optimizar el diagnóstico oportuno de anemia infantil.

\section{Reflexiones finales y recomendaciones}

Uno de los factores de la alta incidencia de anemia en nuestro país es la falta de conocimiento sobre las causas y consecuencias de la anemia ${ }^{[23]}$. En ese sentido, se debe considerar la efectividad de usar tecnologías de la información y comunicación como medios alternativos a los medios de comunicación tradicionales (como radio, TV y prensa) para la difusión de mensajes educativos en la población.

Es importante mencionar que existen pocos estudios de costoutilidad y costo-efectividad para sistemas de salud electrónica y salud móvil en la literatura. Algunos estudios de costo-efectividad demuestran que la telemedicina puede reducir los costos, pero no todos. Entre las principales limitaciones de las evaluaciones económicas de los sistemas de telemedicina se encuentran la falta de estudios controlados y aleatorizados, el tamaño pequeño de las muestras, y la poca calidad de los datos ${ }^{[24]}$. Se recomienda que en futuras revisiones sobre el tema, incluyan las perspectivas de impacto económico (costo-utilidad y costoefectividad) de aplicaciones de salud móvil en la lucha contra la anemia considerando distintos escenarios.

En los estudios revisados en este artículo se observó que el uso de SMS para difusión de información cuenta con una serie de ventajas, entre las que destaca su bajo costo, su facilidad de uso, acceso, y disponibilidad. Además de ello, se observó un incremento en los niveles de adherencia al tratamiento, disminución de citas no asistidas y aumento de asistencia a citas médicas. En el caso de mensajes recordatorios y motivacionales sobre la anemia se puede evaluar la viabilidad de enviar dos tipos de mensajes: mensajes recordatorios para asistencia a controles de crecimiento y desarrollo (con frecuencia variable dependiendo de la edad del niño) y mensajes motivacionales sobre la importancia del consumo de alimentos ricos en hierro.

En el caso del uso de celulares como mecanismo a de sensibilización e información para los ciudadanos, se recomienda que los mensajes deben ser claros y culturalmente adaptados para la población, utilizando no sólo imágenes o videos, sino incluso potenciados con el despliegue de campañas por radio y televisión para la difusión de mensajes de prevención de anemia.

Otra recomendación planteada seria el explorar el uso de tecnologías móviles en los agentes de salud comunitarios para gestionar información, para capacitación de los recursos humanos, y fortalecer las redes de contacto y comunicación con los servicios de salud y las autoridades.

En conclusión, se conoce que la anemia es un problema multicausal y existe evidencia sobre de intervenciones eficaces que utilizan las TICs; interviniendo mediante el uso de teléfonos celulares incluyendo los mensajes de texto. Estas estrategias, al ser económicas y de fácil acceso-comprensión para el usuario, se deberían considerar dentro de las estrategias en la lucha contra la anemia a nivel nacional. 
Es recomendable evaluar la combinación del envío de los mensajes de texto con otras intervenciones como las visitas domiciliarias o talleres. Asimismo, es importante potenciar los portales web y un observatorio multisectorial de anemia que permitan el seguimiento efectivo de las acciones en el marco del Plan Multisectorial de Lucha contra la Anemia y que sirvan como instrumento de gestión efectivos para las instancias de gobiernos locales y regionales.

Sin embargo, existe una gran necesidad de más estudios que generen evidencia necesaria para incorporar las herramientas basadas en teléfonos celulares como parte de la política y acciones multisectoriales e intergubernamentales en la lucha contra la anemia.

Contribución de los autores: EEP, MHS y GJVC participaron en la redacción del manuscrito y aprobaron la versión final a publicar.

\section{Fuentes de financiamiento: Autofinanciado.}

Conflicto de interés: Los autores declaran no tener conflictos de interés en la publicación de este artículo.

\section{REFERENCIAS BIBLIOGRÁFICAS}

1. Banco Mundial. Prevalencia de anemia en la infancia (\% de menores de 5 años) [Internet]. Washington, DC: Banco Mundial; 2016 [citado el 2 de noviembre de 2018]. Disponible en: https://datos. bancomundial.org/indicador/SH.ANM.CHLD.ZS

2. Instituto Nacional de Estadística e Informática. Perú: Indicadores de Resultados de los Programas Presupuestales 2013-2018. Encuesta Demográfica y de Salud Familiar [Internet]. Lima: INEI; 2019 [citado el 2 de noviembre de 2018]. Disponible en: https://proyectos. inei.gob.pe/endes/2018/ppr_2013_2018/Indicadores\%20de\%20 Resultados\%20de\%20los\%20Programas\%20Presupuestales_ ENDES_2018.pdf

3. Comisión Interministerial de Asuntos Sociales. Plan Multisectorial de Lucha contra la Anemia [Internet]. Lima: Ministerio de Desarrollo e Inclusión Social; 2016 [citado el 2 de noviembre de 2018]. Disponible en: http://www.midis.gob.pe/dmdocuments/planmultisectorial-de-lucha-contra-la-anemia-v3.pdf

4. Curioso WH. eSalud en Perú: implementación de políticas para el fortalecimiento de sistemas de información en salud. Rev Panam Salud Pública. 2014;35:437-41.

5. Instituto Nacional de Estadística e Informática. Perú: Perfil Sociodemográfico. Informe Nacional de los Censos Nacionales 2017: XII de Población, VII de Vivienda y III de Comunidades Indígenas. Lima: Instituto Nacional de Estadística e Informática; 2018.

6. Instituto Nacional de Estadística e Informática. Censos Nacionales 2007: XI de Población y VI de Vivienda. Perú: Crecimiento y distribución de la población, 2007. Lima: INEl; 2008.

7. Ruiz EF, Proaño Á, Ponce OJ, Curioso WH. Tecnologías móviles para la salud pública en el Perú: lecciones aprendidas. Rev Peru Med Exp Salud Publica. 2015;32(2):264-72.

8. Curioso WH. La Telesalud y las nuevas fronteras de la informática biomédica en el Perú. Rev Peru Med Exp Salud Publica. 2015;32(2):217-20.

9. Tomlinson M, Rotheram-Borus MJ, Swartz L, Tsai AC. Scaling Up mHealth: Where Is the Evidence? PLoS Med. 2013;10(2):e1001382.
10. Kannisto KA, Koivunen MH, Välimäki MA. Use of mobile phone text message reminders in health care services: a narrative literature review. J Med Internet Res. 2014;16(10):e222.

11. Lee SH, Nurmatov UB, Nwaru BI, Mukherjee M, Grant L, Pagliari C. Effectiveness of mHealth interventions for maternal, newborn and child health in low-and middle-income countries: Systematic review and meta-analysis. J Glob Health. 2019;6(1):010401.

12. Duan $V$, Jia W, Pang $X, B i Y$, Wang J, Yin S, et al. Effects of text message intervention on infant growth and anemia at 6 month old. Wei Sheng Yan Jiu. 2016;45(2):226-9, 235.

13. Zhou H, Sun S, Luo R, Sylvia S, Yue A, Shi Y, et al. Impact of Text Message Reminders on Caregivers' Adherence to a Home Fortification Program Against Child Anemia in Rural Western China: A Cluster-Randomized Controlled Trial. Am J Public Health. 2016;106(7):1256-62.

14. Khorshid MR, Afshari P, Abedi P. The effect of SMS messaging on the compliance with iron supplementation among pregnant women in Iran: a randomized controlled trial. J Telemed Telecare. 2014;20(4):201-6.

15. Rounseville M, Gutierrez N, Oviedo A, Cuevas F, Perochena-Meza G, Medina C, et al. Texting for Nutrition Project and Impact Evaluation. Washington, DC: The World Bank; 2017.

16. Ministerio de Desarrollo e Inclusión Social. Piloto de envío de SMS para reducir la prevalencia de anemia en los hogares usuarios del programa JUNTOS - Nota Metodológica [Internet]. Lima: MIDIS; 2018 [citado el 2 de noviembre de 2018]. Disponible en: http:// evidencia.midis.gob.pe/piloto-de-envio-de-sms-para-reducir-laprevalencia-de-anemia-en-los-hogares-usuarios-del-programajuntos-nota-metodologica/

17. Mannino RG, Myers DR, Tyburski EA, Caruso C, Boudreaux J, Leong $T$, et al. Smartphone app for non-invasive detection of anemia using only patient-sourced photos. Nat Commun. 2018;9(1):4924.

18. El Peruano. Aplicación móvil para prevenir la anemia [Internet]. Lima: El Peruano; 2018 [citado el 2 de noviembre de 2018]. Disponible en: $h$ ttp://elperuano.pe/noticia-aplicacion-movil-paraprevenir-anemia-72280.aspx

19. Editora Perú. Lucha contra la anemia: Aplicativo móvil ayudará a prevenir y controlar enfermedad [Internet]. Lima: Agencia Peruana de Noticias; 2018 [citado el 2 de noviembre de 2018]. Disponible en: https://andina.pe/agencia/noticia-lucha-contra-anemia-aplicativomovil-ayudara-a-prevenir-y-controlar-enfermedad-729664.aspx

20. Breslauer DN, Maamari RN, Switz NA, Lam WA, Fletcher DA. Mobile Phone Based Clinical Microscopy for Global Health Applications. Plos One. 2009;4(7):e6320.

21. Ministerio de Desarrollo e Inclusión Social. AYNI Lab [Internet]. Lima: MIDIS; 2018 [citado el 2 de noviembre de 2018]. Disponible en: http://evidencia.midis.gob.pe/ayni-lab/

22. Ministerio de Desarrollo e Inclusión Social, Universidad Peruana Cayetano Heredia, Universidad de Ciencias y Humanidades. Sistema portátil para el diagnóstico de anemia basado en el análisis de la conjuntiva ocular usando un smartphone e inteligencia artificial [Internet]. Lima: MIDIS; 2018 [citado el 2 de noviembre de 2018]. Disponible en: http://evidencia.midis.gob.pe/wp-content/ uploads/2018/05/FICHA-RESUMEN-UPCH-Smartphone.pdf

23. Aparco JP, Huamán-Espino L. Barreras y facilitadores a la suplementación con micronutrientes en polvo: percepciones maternas y dinámica de los servicios de salud. Rev Peru Med Exp Salud Publica. 2017;34(4):590-600.

24. de la Torre-Díez I, López-Coronado M, Vaca C, Aguado JS, de Castro C. Cost-utility and cost-effectiveness studies of telemedicine, electronic, and mobile health systems in the literature: a systematic review. Telemed J E-Health. 2015;21(2):81-5. 